\title{
Stress, Psychoneuroimmunology and Self-care: What Every Nurse Needs to Know
}

\section{Carey S Clark ${ }^{*}$}

Assistant Professor, University of Maine At Augusta, USA

*Corresponding author: Carey S Clark, Phd, RN, AHN-BC, Assistant Professor, University of Maine At Augusta, USA, Tel: 707-239-6738; E-mail: Carey.clark@maine.edu

Received date: Oct 14, 2013, Accepted date: Feb 06, 2014, Published date: March 10, 2014

Copyright: (c) 2014 Carey S Clark, This is an open-access article distributed under the terms of the Creative Commons Attribution License, which permits unrestricted use, distribution, and reproduction in any medium, provided the original author and source are credited.

\begin{abstract}
This paper explores the effects of stress on nurses, patients, and the healing environments. Through a synthesized review of the effects of stress on the psychoneuro-immunological system, this article provides strong evidence for why nurses must create and enact caring-healing processes from both a physiological and ethical perspective. The ethical call toward decreasing stress for the self, for our patients, and in our workplace environments is made.
\end{abstract}

Keywords: Caring; Healing; Ethics; Nursing; Stress; Methods

Psychoneuroimmunology; Self-care

\section{Stress, Psychoneuroimmunology, and Self-care: What Every Nurse Needs to Know}

Most nurses come to the profession with a deep desire to support the healing process of others, knowing that this is the rewarding aspect of the work nurses do; and yet many nurses find that they are underequipped in both academia and professional healthcare settings to engage and support patients in their healing processes [1]. Nurses are charged with caring for people during their most vulnerable times of illness, as well as supporting all persons in maintaining or improving their wellness status. As our educational and practice settings have become overly-medicalized, the profession and the systems in which nurse's work have failed to support nurses in their own self-care and healing practices. Nurses must role model wellness, strive toward supporting others in their wellness, and create caring-healing bedside practices that are sustainable; the current systems do not enable nurses to enact our guiding caring theories and uphold nursing's ethical standards [2,3]. Likewise, nurses work environments are mired in medical-technical demands, and the environments have become so stress inducing, that the process of creating caring-healing environments seems unattainable to many. The stress of professional responsibilities and the inability of nurses to create the caring-healing environment contribute to the ongoing costly issues as one considers low new graduate nurse retention rates and the issue of nurse burnout $[2,3]$.

This paper addresses the psychoneuro-immunological implications of stress, for both the patient and the nurse. As well, the paper creates a call for the implementation of nurses' daily self-care and healing strategies to transform our academic settings and healthcare workplaces into sustainable caring-healing environments. Concepts explored include the stress response, secondary stress syndrome, telomeres, mirror neurons, neuroplasticity, mind-body medicine, the relaxation response, and a call for change.
A literature review process was used in collecting the data for this paper. The writer started by reviewing numerous textbooks on stress and psychoneuroimmunology to determine an overall consensus regarding the current concept of stress as related to the contemporary healthcare system and stress-based disease processes. Several of these texts were found via Google search engine and amazon.com platforms. Additionally, the Cumulative Index of Nursing and Allied Health Literature (CINAHL) database was used to find supportive literature related to the overall goal of the paper, namely synthesizing the evidence around stress based research and sharing with nurses the implications of stress in the workplace. The primary purpose of this process was to "provide the reader with a comprehensive background for understanding current knowledge and highlighting the significance of new research" [3].

\section{Overviews and Brief Definitions: Stress, Psychoneuroimmunology and Allostatic Load}

Anywhere from $60-90 \%$ of primary care visits are stress related [4], and this will have a huge impact on the healthcare field as providers begin to look for ways to better manage the influx of patients with the healthcare system with the Patient Affordable Care Act implementation on a grand scale. While most nurses have some familiarity with the term stress and the physiology of the fight or flight response, there may be some lack of knowledge around the everyday contemporary stress response, which seems to be based more in psychological perceptions versus the many physiological threats our ancestors faced. It is helpful to have a brief understanding of the terms like psychoneuroimmunology and allostatic load before proceeding with more depth regarding stress, how it creates a healthcare environment that is not conducive to healing, and how stress may indeed favor the continuation of the disease-illness process.

\section{Stress: Definitions}

While there may be many definitions of stress, for purposes of this paper, stress will be defined as, "A particular relationship between the 
person and the environment that is appraised by the person as taxing or exceeding his or her resources and endangering his or her wellbeing" [5]. When we examine contemporary stress, it is often based in psychological factors around the perception of a threatening event in the environment, whether that particular threat is "real" or not. These psychological perceptions of stress are also based in our prior experiences, and the amygdala and limbic systems scan the environment for these stress triggers on a regular basis [6]. Meanwhile physiological stress in contemporary times (which may be related to exposure to toxins, dietary exposures that set off the inflammatory response, lack of sleep, and lifestyle habits) leads to the stress response being initiated in the body's brain. Primarily the lower brain areas of the amygdala and limbic system and subsequent activation of the hypothalamic-pituitary adrenal system are of concern. When the person perceives a stressor, there is a subsequent release of hormones such as cortisol (which helps to organize body system responses), as well as neurotransmitters, including the catecholamines, dopamine, epinephrine, and nor-epinephrine [6]. While this alarm stage prepares the body for "fight or flight" scenarios, it also causes great damage to the cells in our body, particularly with persistent stress states where the body is staying aroused for long periods or on a frequent basis. With frequent repetition of the stress state and lack of time for recovery, adrenal fatigue and maladaptation can occur [6]. Even mild to moderate chronic stress damages tissues and organs, and has been related to high blood pressure, heart disease, rheumatoid arthritis, and many other illness states related to suppression of the immune system $[6,7]$. Stress also impacts the hippocampus section of the brain in such a way that thinking and memory become impaired. With prolonged stress states, the prefrontal cortex dendrites of the brain are damaged, decreasing the person's ability to make higher ordered decisions and undertake critical thinking processes; it should be noted that young people's developing brains are particularly vulnerable to stress [7].

The activation of stress in our bodies is different for each of us and is related to how our brains formed in childhood, including our stress resilience and the number of adverse childhood events we experienced. While not all stress is negative (a small amount of stress helps to motivate an individual to relieve stress and move back toward allostasis), toxic and chronic stress levels (where adverse stress is experienced on an ongoing or prolonged basis) and the resultant high levels of cortisol can lead to permanent changes in the brain, creating brains that are less resilient to the effects of stress. Stress also impacts the immune system and capacity for learning [7].

\section{Allostatic load}

Allostatic load (AL) is a term used to describe the cumulative physiological effects of the body's efforts to adapt to continuing stress over time [8]. The following is a clear definition of the term.

Allostasis emphasizes the dynamic, nonlinear nature of a host of biological mediators that are turned on by stressors in order to maintain homeostasis and promote adaptation. The social environment in which we live is an enormous source of challenges that require allostasis. Problems arise, however, if the allostatic response to a stressor is not turned on when needed or not turned off when the stressor is over. In these cases, the body and brain experience negative consequences that, over time and repetition of stressors, may accumulate and result in pathophysiology. This is allostatic load or overload in the extreme [9].

$\mathrm{AL}$ is a sign of aging related to stress and the higher the $\mathrm{AL}$, the more likely one is to become ill and struggle with healing. AL has been translated into a measurable index, and it is used to gauge the physiological effects of chronic stress in the workplace; high physiological stress and low job control, have been shown to be a risk factor for a higher AL and multiple system dysfunctions [10], as well as chronic critical illness syndrome [11]. Additionally, as one ages, the effects of stress on AL seem to increase, particularly after the age of 45 . As of this date, studies have not been done on the AL of nurses in particular. We may assume that nurses have high stress and the capacity for AL, related to workplace high physiological demands (physical labor), low control, managing of human suffering, long work hours, staffing issues, interpersonal work relationships, increased use of technology, and the aging process of the nurses themselves [10]. AL becomes a bigger concern for nurses as we age, as stress seems to more readily lead to $\mathrm{AL}$ as the body ages, noticeably increasing after the age of 45 .

\section{Psychoneuroimmunology}

Psychoneuroimmunology (PNI) is the trans disciplinary field of study concerned with how behavior, the central nervous system, the endocrine system, and the immune system interact; the field examines how consciousness impacts one's central nervous system and immune systems, and examines the complex flow of information across body systems [11]. PNI is focused on this interaction of behaviors, emotions, psychological responses, the central nervous system, and the immune system, and how these interactions can lead to illness or wellness. The following definition proves helpful when discussing this field of study:

"Psychoneuroimmunology, a maturing science that investigates connections among stress, Emotions, neurochemistry and immunity at every level from molecular to ecosystem. The immune system is a very sensitive index of successful or unsuccessful allostasis since acute stress will enhance immune defenses, while chronic stress can suppress those defenses. Moreover, inflammatory processes are part of all the major disorders of modern life-from diabetes to cancer to neurodegenerative brain diseases-and the regulation of inflammation involves not only glucocorticoids but also sympathetic/ parasympathetic balance" [9].

PNI is important to nurses because we guide people to move through suffering and toward wellness and health; without understanding what is immune supportive and immunosuppressant, and how the body works together integrally through multiple body systems or a whole persona response, the nurse lacks some key foundational concepts on how to support others in their own wellness and healing progression [12]. Unfortunately, the whole body systems or complexity view of the body, illness, and wellness are generally not focused on in our academic and professional experiences as nurses [13].

People experiencing illness are experiencing stress; meanwhile the experience of seeking healthcare and hospitalization may serve to increase and compound stress levels. How nurses physiologically and emotionally show up to interact with patients either supports the patients maintaining or worsening stress levels and the inherent detrimental effects, or facilitates their movement toward relaxation and healing. The following section will review nurses' stress levels, how the nurse's stress impacts the patient's stress, and healing capacity. 


\section{Nurses Stress: A Review of the Issues in the Literature}

Stress has been greatly studied in the healthcare workplace and with nurses in particular, there have been many efforts made to measure, analyze, and address the stress nurses face. Nurse's stress in the workplace was first recognized in the 1950's and it remains a cause of concern today [14]. As the review below indicates, nurses feel and experience ongoing physiological and psychological stress.

\section{Stress and the workplace demands}

Nurses workplace stress is a related to emotional exhaustion, workplace conditions, continually dealing with death and dying, work control issues, long working hours, poor recovery from working shifts with limited sleep opportunity, rapid work pace, lack of support, working weekends with less support, nurse staffing issues, lack of nurse management involvement, lack of empowerment, job ambiguity, and job interference with family needs and concerns [15]. Stress levels of nurses may be related to length of time employed: nurses who have less experience and are not yet at expert levels of providing care report greater levels of stress, likely related to challenges with organization, interruptions, anticipating patient needs, communicating with patients and staff, and a deeper integration of the role of the nurse [16]. Additionally, there may be role specific stressors with some variance for nurse managers versus other roles, such as that of the charge nurse and the floor nurse [17].

Of particular concern is the sheer amount of time that nurses spend working, which in general far exceeds what the Institutes of Medicine recommends [18]. For instance, when examining the work schedule of

\section{2, 273 nurses, [18] found that:}

More than $25 \%$ of nurses work more than 12 hours per day

$50 \%$ of hospital nurses work more than 12 hours/day

More than $30 \%$ of nurses worked more than 40 hours/week, and on call requirements are required of nearly $40 \%$ of all nurses.

Couple these working hours with very little time off between shifts, and there is a clear recipe for physiological stress, which impacts the likelihood of nurses' poor health and increases the risks for making errors.

\section{Critical incident stress or secondary stress syndrome}

Nurses are exposed to patients who experience severe traumatic incidents, and though the nurse is not likely present during the actual traumatic event, the effects of being close to the people involved in the incident can lead to a stress like response in the body similar to post traumatic stress disorder [19]. One study found that this rate of experiencing secondary trauma amongst general hospital setting nurses varies from a low of thirty percent to as high as ninety percent [20]. Nurses exposed to secondary trauma may experience insomnia, intrusion, avoidance, and dissociation, re-experiencing of the event, compassion fatigue, burnout, and hyper-arousal [20]. The closer one is to the traumatic event (i.e. walking with a family through a traumatic loss), the more severe the impact may be. Therefore the more involved nurse, who also does not have effective stress management tools, may be more at risk for the effects of critical incident or secondary stress syndrome [19], eventually suffering many of the same physiological impacts of post-traumatic stress disorder.
While it is clear that stress for nurses has been an issue for some decades, and it remains an issue today, what is not clear is what nurses are doing about stress in their personal life and in the workplace. The following sections review the implications of stress for nurses, nursing practice, and the patients we care for.

\section{Nurse Stress: The Patient Impact Inclusive of Telomeres, Neuron-mirroring and Neurophysiology}

Nurse stress impacts not only the quality of safety of care that patients receive; it may also impact the stress levels of patients themselves. This section will provide the theoretical background in support of this idea that nurse stress impacts the patient's own healing capacity and may cause actual physiological harm to the patient whom we seek to care for and support along their healing journey.

\section{Telomeres and stress}

A new area of science and genetics has focused on exploring the function of telomeres; telomeres are the protective nucleotide sequences at the end of chromosomes [21]. The function of the telomere is to protect the chromosome from breaking down or from enacting fusion with neighbouring chromosomes. There is an enzyme called telomerase that helps maintain telomere length, and telomeres also seem to naturally shorten with age due to repetitive cell division, hence many age related diseases are associated with decreased telomere length [21]. Cancer and other illnesses such as cardiovascular disease and diabetes are also associated with shortened telomere length; shortened telomere length is indicative of poor clinical outcomes with disease processes [21,22].

Studies have indicated that leukocyte telomere length is impacted by job stress, and the stress hormone cortisol inhibits the protective factors of the enzyme telomerase, which generally protects telomere length [21]. This is of concern to nurses personally when we think of our own health status, but also for our patients' health status. If we are working in stressful environments, it is likely to be harmfully impacting our patients on this genetic level as they then are embedded in and experience the stress of the healthcare system environment. Our patients may already be coming to us suffering from the effects of stress on the immune system, with shortened telomere length linked to the illness and stress processes, and yet the hospital setting itself may produce unmanageable social and psychological stress which can be as insidious as smoking and fast food to the patient's health status and healing capacity [23].

\section{Post-traumatic stress syndrome post hospitalization}

Posttraumatic stress disorder (PTSD) related to hospitalization is a documented and analyzed phenomenon [24,25], with PTSD symptoms arising in as many as one out of three ICU patients post hospitalization [26]. According to this research performed by Johns Hopkins University, PTSD from an ICU stay is as or more common than PTSD in soldiers, yet little is being done on a broad scale to both recognize and proactively address this concern $[25,27]$.

The Mirror Neuron System: Implications for Communication and Stress

Neuroscience has been able to document how the human brain uses a mechanism to experience empathy and recognize the intention of others through merely observing their behavior and automatically matching the brain activity that correlates with the observed behaviors 
[27]. Communication in nursing is key to successful outcomes for patients. Mirror neuron research helps nurses to understand how communication patterns arising form our brains (the speaker- nurse) are directly related to similar patterns arising in the listener's brain (the patient) if the communication is successful [28]. When we consider mirror neurons in the context of communication, we know that neural pathways of listeners mirror those same pathways as the speaker; hence both speaker and listener evoke the same brain state [28]. However, speaker-listener mirror neural coupling is absent with unsuccessful communication. This neurological action has great implications for how we communicate with, support, and educate our patients.

The concept of mirror neurons provides us with some explanations of how we deeply understand not only another's physical actions or speech, but also how we understand another's mind and their intentions. In other words, on an unconscious neurological level, patients are able to gage the nurse's mind set and their intentions. If the nurse is there to get their pay check and make it through the shift, the patient knows this via neurological wisdom; conversely if the nurse is there with the intention of being a source of caring, compassion, love, and healing for the patient, this is also conveyed. Additionally, the nurse's stress state is easily communicated via mirror neurons to the patient, potentially causing harm as the stress related neural pathways are physiologically mirrored and replicated in the patient.

\section{Emotional attractors}

Brain science to demonstrate that nurses' own emotional state is reflected on to our patients [29]. It is important from a neurophysiological perspective that when we are working with patients, we are considering our own emotional state and levels of stress in relation to the implications for the patients we care for.

The limbic (emotional) brain contains its emotional attractors, encoded early in life. Primal bias then forms an integral part of the neural systems that view the emotional world and conduct relationships. Limbic attractors exert a distorting force not only within the brain that produces them, but also on the limbic networks of others-calling forth compatible memories, emotional states, and styles of relatedness in them. Through the limbic transmission of an attractor's influence, one person can lure others into his or her emotional reality. All of us, when we engage in relatedness, fall under the gravitational influence of another's emotional mind [29,30].

Do we as nurses consider our emotional state when we enter into the room of a patient we are caring for? Is this a practice that is taught in nursing schools and carried forward into the workplace setting? Are we aware of the impact our own emotional state has on the neurons of patients? Can the profession begin to clearly recognize how we, through our own beings, influence healing and potentially the suffering of the patients we serve?

As we become part of the patient's environment, it becomes imperative that we are caring and supportive of decreasing patient's stress, lessening anxiety, and increasing their sense of control [12]. Unfortunately, many nurses themselves are also under stress related to workplace demands, and when the nurse is stressed, not only does he or she suffer the same PNI implications as the patient, the nurse may also be inadvertently supporting the continuing stress response and its negative effects on the patient.

While we have holistic and caring nursing theories to guide our nursing practices, stress impedes our ability to live up to our moral ideal or moral imperative of being in relationship with oneself, others, and the environment in a way that is caring and supportive of the healing process $[1,31]$. It is fair to say that stress-based practice is not how most nurses want to participate in professional nursing, it is likely a grand contributing factor to burnout within the profession, and we need to consider how we can move out of stress response and toward enacting caring-healing modalities.

\section{Stress and the Relaxation Response: Ethical Implications and Becoming the Healing Environment}

The neuro-physiological evidence for the need for nurses to manage stress for their own well-being and the inter-relation to the healing process of their patients has been presented. In many ways, it may be easy for nursing as profession to simply blame the systems in which we work, or to claim lack of emancipation and autonomy as the reasons for why we have no control over the stress response in ourselves and in the environments where we work. However, when one examines the Code of Ethics for Nurses, we can see that the profession's on-going issues with personal stress and stress in the workplace directly relate to our ability to uphold the following ethical provisions:

Provision 3 (The nurse promotes, advocates for, and strives to protect the health, safety, and rights of the patient),

Provision 6 (The nurse participates in establishing, maintaining, and improving health care environments and conditions of employment conducive to the provision of quality health care and consistent with the values of the profession through individual and collective action),

Provision 9 (The profession of nursing, as represented by associations and their members, is responsible for articulating nursing values, for maintaining the integrity of the profession and its practice, and for shaping social policy [31].

Indeed as a profession, nurses are charged with alleviating suffering and restoring health, while also being called to change the social structures that do not support health, healing, and well-being. As per the code:

Nursing encompasses the prevention of illness, the alleviation of suffering, and the protection, promotion, and restoration of health in the care of individuals, families, groups, and communities. Nurses act to change those aspects of social structures that detract from health and well-being. Individuals who become nurses are expected not only to adhere to the ideals and moral norms of the profession but also to embrace them as a part of what it means to be a nurse [31].

In my work as a nurse educator teaching RN- BSN students from an integral- holistic curricular framework, beginning students often assert that the ideas of change and transformative leadership sound great, but nurses really cannot do this in the workplace. Our goal though is to overcome this resistance and support students to make workplace change by first making change within the self. The following sections will outline how each nurse can begin to take self-responsibility for supporting the healing process with self and patients, with the ultimate goal of being able to uphold the Code of Ethics For Nurses [31] and transform healthcare workplaces into places of meaningful healing and personal evolution versus the current norm of stress and illness inducing environments. 


\section{The Relaxation Response and Neuroplasticity: What Every Nurse Needs to Know}

The good news about stress is that there are many tools available for nurses to use to assist us in calming the nervous system and enacting the relaxation response to support healing. Additionally, our brains and nervous systems are neuroplastic, meaning regardless of our age or capacity, our brains are capable of making new neural pathways and our engrained habits and ways of being, given the correct stimulation, can be revised [32]. The issues of the impact of chronic low stress or acute toxic stress, both of which can be highly damaging to the body through the cascade of stress hormones released, can be addressed through daily practice of the relaxation response.

\section{The relaxation response: Top down brain processes}

In the late 1960's, Herbert Benson, an MD out of Harvard Medical School determined that there was a counter-balancing response to the physiological stress response and aptly names the response the relaxation response [33]. Just as the hypothalamus and amygdala are responsible for enacting the stress response, so too there are other parts of the brain responsible for enacting the counter-balancing relaxation response. The relaxation response, which is regulated by the prefrontal cortex, creates physical and emotional responses to stress that lead to decreases in heart rate, blood pressure, respiratory rates, and muscle tension. The prefrontal cortex ("top" of the brain) is also the region of the brain where our higher ordered thinking originates from and techniques to enter the relaxation response, help to support the use of the prefrontal cortex for responding and thinking; through a variety of neurotransmitters such as GABA, the prefrontal cortex directs the amygdala and hypothalamus ("bottom" part of the brain) to move out of the stress response hormone cascade. Therefore a top down brain response, where one is using high ordered thinking and supporting the relaxation response, is preferred to the opposite direction [34].

\section{Relaxation response: Techniques}

Many things can be done to enter into the relaxation response and/ or avoid the stress response, creating optimal brain conditions for a top down response, and prime conditions for enacting caring and healing presence with patients; they are also the same techniques that patients can learn to reduce their stress levels and move toward healing [34]. Whatever methods are used, the nurse needs to enact the relaxation response on a daily basis to begin to create the sort of changes necessary in the self to combat workplace stress, and be able to interact with patients in a caring-healing-supportive manner and in a way that upholds the ethical obligations of the profession.

\section{Meditation \\ Nurses who practice mindfulness-based meditation had an improved sense of psychological well- being, better coherence, and were better prepared to cope with stress [35]. Meditation is an activity that can be done every day to support the relaxation response and also strengthen the anti-oxidant gene expression of an individual [32]. While many people mis-perceive meditation to be the act of emptying the mind, the idea or goal is to create a focus and then observe the mind's activities in an accepting way, accepting and noticing when the mind wanders, and bringing the thoughts gently back to the focus. Benson and Proctor recommend taking 10-20 minutes, 1-2 times/day, before breakfast or before dinner to sit quietly and focus on an internal}

repetition of a word, positive phrase, chant, or prayer. As thoughts arise to distract one from one's focus, an attitude of observation and passive disregard for the emerging thoughts is enacted, and one gently and compassionately notices this pattern and thought and simply returns to the focus of the word, phrase, chant, or prayer. This daily activity has been shown to create neural and genetic marker changes that are health positive and combat the effects of stress [32]. Use of MRI demonstrated structural changes in the brain toward a greater top down response in as little as 8 weeks of daily practice of meditation.

\section{Mindful movement}

Movement with attention may be known as mindful movement and would include such techniques as yoga, tai chi, and qi-gong. Movement with attention creates new neural pathways, and also stimulates the vagus nerve, causing the resting state of muscles to be reinforced [6,36]. Researchers at Ohio State University have determined that regular yoga practice can lower levels of interleukin- 6 in their blood both before and after experiencing a stressful event. High levels of IL- 6 are related to the stress response, the subsequent high inflammatory response by the body and are related to illnesses such as heart disease, stroke, type- 2 diabetes, arthritis and age related debilitating diseases [37]. Indeed, the researchers, in comparing novice yoginis to expert yoginis, also determined that more expert yoginis (greater length of practice) were better able to physiologically limit the stress response; this is important because it points to the long term anti-stress effects of a regular yoga practice. Additionally, physical exercise has long been recognized as a form of moving meditation and it supports relaxation through endorphin release, self-awareness, and spiritual awareness through the body's movements [32].

\section{The good experience and therapeutic tools}

The "good experience exercise" includes the idea of thinking positively or using tools such as cognitive behavioral therapy to reframe one's perception of the stressful triggers; this positive thinking becomes important when one is considering that the contemporary stress response is generally framed around a person's perception of stress [38]. Tools such as bio-neuro feedback, stress journaling, and talk therapy may also serve to change the perception of stress and thereby modify one's physiological response to stress.

\section{Physiological and environmental issues}

One of the most important physiological issues related to stress is sleep; sleep is needed to regenerate the brain cells as shown by MRI [39]. Even partial sleep deprivation leads to stress, with the average sleep requirement to avoid stress and experience rest and renewal being 7-8 hours' sleep/night [40]. Partial sleep deprivation (even just one hour less than the needed 7 hours) can lead to mood regulation issues, cognitive difficulties, decreased job performance, reduced motivation, greater safety risks, and physiological changes, with great implications for the nurse's physiological stress level [41] or AL. Nurses in particular are at risk for the effects of sleep deprivation, and unfortunately lack of sleep and the resultant physiological stress response make us more vulnerable to making errors and risking patient safety:

Nurses regularly short-change themselves on sleep, getting by on an average of 6.8 hours of sleep on their work days instead of the commonly recommended 8 hours per 24 -hour period. On-going sleep 
deprivation of as little as an hour a day can lead to a sleep debt over time that is not easily erased. When we have a sleep debt, our inclination to fall asleep the next day increases. The larger the sleep debt, the stronger the tendency to fall asleep. Sleep debt does not go away by itself. Sleeping is the only way to erase sleep debt [42].

Other physiological triggers of stress include a high inflammatory diet, sugar, alcohol, nicotine, and caffeine, all of which can be managed through lifestyle changes, but are often quite challenging to do in our current cultural environment.

\section{Recommendations}

That nurse's work in stressful environments has not been disputed; what is of concern is how this stress impacts our bodies, minds, and spirits and the greater implications for our patients. As well, nurses need to consider how our own stress levels impact our ability to work well with teams and patients; may we as a profession begin to consider that perhaps the profession of nursing contributes to the stress filled healthcare environments? If we desire to create caring-healing sustainable nursing practices, the evidence presented here points to the need for stress management techniques to be employed by each and every nurse. As mentioned above, as each nurse begins to practice reflection, and enact the relaxation response on a daily basis, nurses can begin to move toward places of compassion for our patients and for ourselves. Without these practices in place, without each and every nurse addressing their own need for self-care, healing, and compassion enhancement, we are destined toward working in this continually broken and ironic or paradoxical system, where suffering patients come to us in need of caring and healing, and we remain unable to support this need for caring from the PNI perspective. We suffer ourselves from the effects of stress and then pass this stress and suffering along to our patients.

With the ideas presented here in mind, it is recommended that schools of nursing and healthcare workplaces begin to support nursing students and practicing nurses in their efforts toward learning about stress management and enacting a self-care-healing journey. As I have reported elsewhere, it is possible to create meaningful nursing curricula that support students to begin enacting self-care that impacts their ability to create sustainable caring-healing environments [43]. Nurses practicing from an integral perspective support the patients healing and evolution through holistic stress management techniques, but nurses also need healthcare facilities to support nurses in learning and utilizing these techniques [43]. Given the evidence presented, every hospital and healthcare system should be vested in decreasing patient and staff stress and creating caring-healing environments.

\section{Conclusion}

The dynamics of relational, human-to-human caring practices and comprehensive therapeutic modalities for caring-healing seem to be eclipsed by the daily routines, mechanics and demands of economic, management, physical and technological aspects of care. The heart of the necessary changes needed for renewal and transformation seem to be dependent on human dimensions and skills that result in transforming patterns and depths of communication, relationships and healing modalities. These human caring-healing dimensions transcend profession, system, and institutional structures [44].

If each and every nurse could realize the importance of the implications for their own wellbeing and the wellbeing of others and the need to strive to uphold the Code of Ethics for Nurses [31], we could begin on a larger scale to change ourselves and the environments in which we work. If we feel called to this work and honored to guide others, each of us has an ethical responsibility to ourselves, our families, and our fellow human companions to change the way we live and decrease our stress in order to become the beacon of hope and healing for ourselves, so that the beacon is passed onto those we care for, the suffering populations. Only through each nurse managing his or her response to stress in efforts to create caringhealing-sustainable practices, will our healthcare systems be transformed toward healing environments and away from the current state of harmful environments secondary to stress implications. While this paper calls for entire healthcare systems to be involved in the process, nurses can also simply take the first steps themselves in learning to enact the relaxation response in an effort to highlight and role model the process for our colleagues, administrators, and patients alike.

\section{References}

1. Clark CS (2010) The nursing shortage as a community transformational opportunity: an update. ANS Adv Nurs Sci 33: 35-52.

2. Clark CS (2002) The nursing shortage as a community transformational opportunity. ANS Adv Nurs Sci 25: 18-31.

3. Cronin P, Ryan F, Coughlan M (2008) Undertaking a literature review: a step-by-step approach. Br J Nurs 17: 38-43.

4. Beth Israel Deaconess Medical Center (2012) Stress management counselling in the primary care setting is rare. ScienceDaily.

5. Lazaurs RS, Folkman S (1984) Stress, appraisal, and coping. New York, NY: Springer.

6. Streeter CC, Gerbarg PL, Saper RB, Ciraulo DA, Brown RP (2012) Effects of yoga on the autonomic nervous system, gamma-aminobutyric-acid and allostasis in epilepsy, depression, and post-traumatic stress disorder. Medical Hypotheses 78: 571-579.

7. Middlebrook JS, Audage NC (2008) The effects of childhood stress on health across the lifespan. Centers for Disease Control and Prevention, National Center for Injury Prevention and Control.

8. Glover DA, Stuber M, Poland RE (2006) Allostatic load in women with and without PTSD symptoms. Psychiatry 69: 191-203.

9. Groer MW, McEwen BS (2012) Nursing research in stress, psychoneuroimmunology, and allostasis. Biol Res Nurs 14: 309-310.

10. Sun J, Weng S, Zhang-J, Li W (2007) Assessing the cumulative effects of stress: The association between job stress and allostatic load in a large sample of Chinese employees. Work \& Stress 21: 333-347.

11. Bellar A, Kunkler K, Burkett M (2009) Understanding, recognizing, and managing chronic critical illness syndrome. J Am Acad Nurse Pract 21: 571-578.

12. Halldorsdottir S (2007) A psychoneuroimmunological view of the healing potential of professional caring in the face of human suffering. International Journal for Human Caring 11: 32-38.

13. Clark CS (2004) Complexity in nursing education: Examples of the paradigm. World Futures 60: 371-388.

14. Authors Jennings BM (2008) Work Stress and Burnout Among Nurses: Role of the Work Environment and Working Conditions. Work Stress and Burnout Among Nurses: Role of the Work Environment and Working Conditions.

15. Purcell SR, Kutash M, Cobb S (2011) The relationship between nurses' stress and nurse staffing factors in a hospital setting. J Nurs Manag 19: 714-720.

16. Burger JL, Parker K, Cason L, Hauck S, Kaetzel D, et al. (2010) Responses to work complexity: the novice to expert effect. West J Nurs Res 32: 497-510.

17. Admi H, Moshe-Eilon Y (2010) Stress among charge nurses: tool development and stress measurement. Nurs Econ 28: 151-158. 
Citation: Clark CS (2014) Stress, Psychoneuroimmunology and Self-care: What Every Nurse Needs to Know. J Nurs Care 3: 146. doi: 10.4172/2167-1168.1000146

Page 7 of 7

18. Trinkoff A, Geiger-Brown J, Brady B, Lipscomb J, Muntaner C (2006) how long and how much are nurses now working? Am J Nurs 106: 60-71.

19. Smith M, Wasilowsky B, Valeriano L (2012) A proactive approach to stress management in a neuroscience intensive care unit. Pa Nurse 67: 12-18.

20. Komachi MH, Kamibeppu K, Nishi D, Matsuoka Y (2012) Secondary traumatic stress and associated factors among Japanese nurses working in hospitals. Int J Nurs Pract 18: 155-163.

21. Ahola K, Sirén I, Kivimäki M, Ripatti S, Aromaa A, et al. (2012) Workrelated exhaustion and telomere length: a population-based study.

22. Ornish D, Lin J, Daubenmier J, Weidner G, Epel E, et al. (2008) Increased telomerase activity and comprehensive lifestyle changes: a pilot study. Lancet Oncol 9: 1048-1057.

23. Blackburn EH, Epel ES (2012) Telomeres and adversity: Too toxic to ignore. Nature 490: 169-171.

24. Daviss WB, Mooney D, Racusin R, Ford JD, Fleischer A, et al. (2000) Predicting posttraumatic stress after hospitalization for pediatric injury. J Am Acad Child Adolesc Psychiatry 39: 576-583.

25. Johns Hopkins Medicine (2013) News and Publications: PTSD symptoms common among ICU survivors.

26. Siddique H (2013) Post Traumatic Stress Disorder (PTSD). Indep Review 15: 107-131.

27. Rossi EL, Rossi KL (2006) The neuroscience of observing consciousness \& mirror neurons in therapeutic hypnosis. Am J Clin Hypn 48: 263-278.

28. Stephens GJ, Silbert LJ, Hasson U (2010) Speaker-listener neural coupling underlies successful communication. Proc Natl Acad Sci U S A 107: $14425-14430$

29. Curtin L (2011) Quantum physics and collegial relationships. American Nurse Today 6: 72 .

30. Cara C (2003) A pragmatic view of Jean Watson's Caring Theory. International Association Journal for Human Caring 7: 5-61.

31. DeWolf Bosek MS, Jannette J, Rambur B (2013) Attitudes of nurses toward patient-directed dying: a pilot study. JONAS Healthc Law Ethics Regul 15: 135-139.

32. Benson H, Proctor W (2011) The relaxation response: The science and genetics of mind body healing. New York, NY: Scribner.
33. Benson Henry Institute for Mind Body Medicine (2013) The Relaxation Response.

34. Begley S (2007) How the brain rewires itself. Time 169: 72-79.

35. Ando M, Natsume T, Kukihara H, Shibata H, Ito S (2011) Efficacy of mindfulness based meditation therapy on the sense of coherence and mental health of nurses. Health , 3: 118-122.

36. Hölzel BK, Carmody J, Vangel M, Congleton C, Yerramsetti SM, et al. (2011) Mindfulness practice leads to increases in regional brain gray matter density. Psychiatry Res 191: 36-43.

37. Kiecolt-Glaser JK, Christian L, Preston H, Houts CR, Malarkey WB, et al. (2010) Stress, inflammation, and yoga practice. Psychosom Med 72: 113-121.

38. Mayo Clinic (2013) Positive thinking: Reduce stress by eliminating negative self-talk.

39. Walker MP, Stickgold R, Alsop D, Gaab N, Schlaug G (2005) Sleepdependent motor memory plasticity in the human brain. Neuroscience 133: 911-917.

40. Dinges DF, Pack F, Williams K, Gillen KA, Powell JW, et al. (1997) Cumulative sleepiness, mood disturbance, and psychomotor vigilance performance decrements during a week of sleep restricted to 4-5 hours per night. Sleep 20: 267-277.

41. Authors Rogers AE (2008) The Effects of Fatigue and Sleepiness on Nurse Performance and Patient Safety. The Effects of Fatigue and Sleepiness on Nurse Performance and Patient Safety .

42. Stowkowski LA (2004) A wake-up call for nurses: Sleep loss, safety, and health. Medscape Nurses Education.

43. Clark CS (2012) Beyond holism: incorporating an integral approach to support caring-healing-sustainable nursing practices. Holist Nurs Pract 26: $92-102$.

44. Watson MJ, Foster R (2003) The Attending Nurse Caring Model: Integrating theory, evidence, and advanced caring-healing therapeutics for transforming professional practice. Journal of Clinical Nursing 12: 360-365. 